\title{
Observations on Ethical Issues in the Neuro-ICU
}

\author{
James L. Bernat ${ }^{1,2}$
}

Published online: 18 April 2015

(c) Springer Science+Business Media New York 2015

In this issue of Neurocritical Care, Souter et al. from the Neurocritical Care Society report their recommendations for optimizing the management of patients with devastating brain injuries [1]. They analyzed evidence and expert opinion to formulate best practices for several aspects of neuro-ICU care. Their guidance likely will inform the standard of medical care of these patients for at least the remainder of this decade.

As a practicing neurologist with a career-long interest in these topics, I offer brief commentaries on four of the ethical issues they discussed: (1) improving prognostic accuracy; (2) improving surrogate decision-making; (3) incorporating principles of palliative medicine into ICU care; and (4) avoiding conflicts in organ donation. I have considered the context of these issues elsewhere in greater detail [2].

\section{Improving Prognostic Accuracy}

It is a truism supported by evidence that prognosis is the cornerstone of medical decision-making [3]. Nowhere is this maxim more apparent than in the patient with a devastating brain injury. If an evidence-based prognosis offers reasonable hope for improvement, most patients and their lawful surrogate decision-makers will decide to pursue aggressive neuro-ICU therapies followed by intensive

James L. Bernat

bernat@dartmouth.edu

Geisel School of Medicine at Dartmouth, Hanover, NH, USA

2 Neurology Department, Dartmouth-Hitchcock Medical Center, Lebanon, NH 03756, USA neurorehabilitation. Conversely, when the prognosis for neurological recovery is poor, patients and surrogates usually choose purely palliative goals. Therefore, arriving at an accurate prognosis is a prerequisite for ethical decision-making [4].

Making and effectively communicating an accurate neurological prognosis is an ethical duty as well as a medical responsibility. Evidence-based neurological prognoses are constrained by several factors. The most common limitation is that many clinical decisions lack an adequate database. Further, the quality of many published studies is degraded by flaws in their design, such as retrospective studies that introduce biases by failing to control independent variables, case-selection bias, absence of confidence intervals, and inadequate powering from a small number of cases. Generalizability of prognostic data may be limited by idiosyncratic practices within institutions and a lack of external validation, both common features of retrospective studies. Unless outcome studies ensure both accuracy and generalizability, they should not be used to determine prognosis [5].

Another limitation in communicating prognosis results from its intrinsic statistical nature. Even when ideal outcome studies have been performed and reported, clinicians often can state a prognosis only statistically. In an adequately powered study, the mean outcome of a group of similar patients can be estimated with reasonable accuracy but there is no way to determine precisely where a given patient will fall within the probability distribution. Therefore, the most accurate rendition (though one that is rarely satisfying to family members) is a statistical prognosis expressed in standard deviations from the mean [6].

The greatest shortcoming of prognostic accuracy in neuro-ICU patients stems from the error of relying on 
experiential studies that fail to control for independent variables. Because asserting prognosis using these studies yields a predictably poor outcome, this error has been called the fallacy of the self-fulfilling prophecy [7]. It has been reported most often in two common neuro-ICU conditions: massive intracerebral hemorrhage [8] and coma or vegetative state after cardiac arrest [7].

The fallacy arises when an unwary physician relies on published experiential outcome data to pronounce a prognosis despite the fact that many or most patients comprising the database were not treated with maximal life-sustaining therapy (LST). Because of their ostensibly poor prognosis, many patients in the studies were allowed to die by DNR orders or orders to withdraw LST. These decisions may have constituted proper medical care for individual patients, but including them in purported "outcome data" makes bad science because it introduces a powerful bias toward a poor outcome. As Becker and colleagues first showed in patients with massive intracerebral hemorrhage [8], DNR orders and orders to reduce LST early in the patient's course led to the self-fulfilling prophecy of death. As revealed in the studies summarized by Souter et al. [1], withholding or withdrawing LST became the most common cause of death in these patients. I concur with their advice to delay decisions to withdraw LST until $72 \mathrm{~h}$ have elapsed (except in the most extreme cases) to allow a determination of clinical response.

\section{Improving Surrogate Decision-Making}

Clinical decision-making for patients with devastating brain injuries nearly always requires a lawful surrogate to consent or refuse therapies on behalf of the patient. Ethical and legal standards for surrogate decision-making require surrogates to try to follow patients' treatment preferences to the fullest extent known. This duty entails following valid written or oral directives previously made by the patient and striving to use the patient's own preferences to determine the goals of therapy. Surrogate decision-making is a lonely and stressful role that requires a good working relationship with the physician, the provision of understandable information, and emotional support [9].

An essential role of the physician is to communicate effectively with the surrogate by providing a realistic view of the diagnosis and prognosis to try to determine the goals of therapy. Although emphasis rightly has been placed on respecting patient autonomy by following the known preferences of the patient, physicians also have an ethical duty to beneficently guide the surrogate to make the best decision [10]. Experienced physicians know that how they frame the discussion with a surrogate influences the response [11]. Surrogates should be instructed that the right decision respects the patient's preference which may differ from what the surrogate would decide for herself.

When a physician senses that a surrogate's guilt impedes the surrogate's ability to reach the right decision, the physician needs to redirect the surrogate. For example, when faced with a poor prognosis some surrogates say, "I know he would not want to be kept alive in this condition but I would always feel guilty if he died because I said to stop LST." Here, it may be helpful for physicians to clarify that they have decided that the best treatment is to withdraw LST and that they plan to order it. This "directive role" framing strategy gently transfers the responsibility of decision-making from the surrogate to the physician and thereby lessens surrogate guilt because the surrogate is being asked only to agree with the physicians' treatment plan, not to choose it [12].

\section{Incorporating Principles of Palliative Medicine}

The field of palliative medicine that initially addressed the needs of dying patients and later incorporated those of chronically ill patients has most recently emphasized its role in the care of critically ill ICU patients. Studies in ICUs show a growing number of patients who fulfill established criteria for palliative care consultations [13]. Several medical centers have successfully incorporated palliative medicine consultations in ICUs and neuro-ICUs. To some extent, the delay in incorporating palliative medicine into ICUs resulted not from an absence of need but from retaining an outdated concept that medical care had to be either aggressive curative or palliative. There is no reason that both goals cannot be achieved.

Palliative medicine offers a holistic approach to the goals of care emphasizing the quality of the patient's remaining life. It incorporates meticulous symptom management, attention to the psychological, emotional, and spiritual needs of the patient, considering the patient embedded within the family, and emphasizes communication and planning. In the neuro-ICU, it has been shown to alleviate physical and emotional symptoms, improve communication, and provide support for patients and families [14]. Experienced palliative medicine consultants have produced tables of practical, specific interventions, considerations, and goals for implementing palliative care in the ICU [15].

\section{Avoiding Conflicts in Organ Donation}

Most neurocritical care physicians have observed instances in which the ethical and legal duty to offer organ donation to families of dying patients has produced conflicts, sometimes when a family member believes that a physician 
has more interest in the patient's organs than in the patient's welfare [16]. Conflicts with the staff and family also have been incited by the relatively new requirement for pre-mortem surveillance by organ procurement organization (OPO) personnel to monitor the progression of an incipiently dying patient to brain death or to the point of withdrawal of LST and potential organ donation after the circulatory determination of death. In their conversations with family members to encourage consent for organ donation, some OPO personnel have adopted a "presumptive approach" that frames the discussion in a leading way that has been off-putting to some physicians and nurses [17].

These apparent conflicts between the duty to care for the dying patient and to offer organ donation can be minimized if critical care physicians and nurses espouse an attitude of "dual advocacy" in which they simultaneously promote excellent end-of-life care and organ donation. The dual advocacy approach is founded on the belief that "given the opportunity, most people will choose to help others" which is the basis of our successful program of voluntary organ donation [18]. Physicians have a beneficence-based ethical duty (and a legal requirement) to offer organ donation to families of deceased patients. Organ donation remains an important medical and social goal that should be supported fully because of the obvious good it provides to the recipients and the associated benefit to the donor family. Each institution needs to develop procedures to optimally integrate OPO personnel to prevent improper intrusion into medical care and to ensure excellent end-of-life management of potential organ donors in the neuro-ICU.

\section{References}

1. Souter MJ, Blissitt PA, Blosser S, et al. Recommendations for the critical care management of devastating brain injury: prognostication, psychosocial and ethical management. A position statement for healthcare professionals from the Neurocritical Care Society. Neurocrit Care. 2015. doi:10.1007/s12028-0150137-6.

2. Bernat JL. Ethical Issues in Neurology. 3rd ed. Philadelphia: Lippincott Williams \& Wilkins; 2008.

3. Murray LS, Teasdale GM, Murray GD, et al. Does prediction of outcome alter patient management? Lancet. 1993;341:1487-91.

4. Bernat JL. Ethical aspects of determining and communicating prognosis in critical care. Neurocrit Care. 2004;1:107-17.

5. Justice AC, Covinsky KE, Berlin JA. Assessing the generalizability of prognostic information. Arch Intern Med. 1999;130: 515-24.

6. Braitman LE, Davidoff F. Predicting clinical states in individual patients. Ann Intern Med. 1996;125:406-12.

7. Shewmon DA, De Giorgio CM. Early prognosis in anoxic coma: reliability and rationale. Neurol Clin. 1989;7:823-43.

8. Becker KJ, Baxter AB, Cohen WA, et al. Withdrawal of support in intracerebral hemorrhage may lead to self-fulfilling prophecies. Neurology. 2001;56:766-72.

9. Vig EK, Starks H, Taylor JS, Hopley EK, Fryer-Edwards K. Surviving surrogate decision-making: what helps and hampers the experience of making medical decisions for others. J Gen Intern Med. 2007;22:1274-9.

10. Ingelfinger F. Arrogance. N Engl J Med. 1980;303:1507-11.

11. Tversky A, Kahneman D. The framing of decisions and the psychology of choice. Science. 1981;211:453-8.

12. White DB, Malvar G, Karr J, Lo B, Curtis JR. Expanding the paradigm of the physician's role in surrogate decision-making: an empirically derived network. Crit Care Med. 2010;38:743-50.

13. Hua M, Wunsch H. Integrating palliative care in the ICU. Curr Opin Crit Care. 2014;20:673-80.

14. Aslakson RA, Curtis JR, Nelson JE. The changing role of palliative care in the ICU. Crit Care Med. 2014;42:2418-28.

15. Cook D, Rocker G. Dying with dignity in the intensive care unit. N Engl J Med. 2014;370:2506-14.

16. Truog RD. Consent for organ donation-balancing conflicting ethical obligations. N Engl J Med. 2008;358:1209-11.

17. Zink S, Wertleib S. A study of the presumptive approach to consent for organ donation: a new solution to an old problem. Crit Care Nurse. 2006;26:129-36.

18. Luskin RS, Glazier AK, Delmonico FL. Organ donation and dual advocacy. N Engl J Med. 2008;358:1207-8. 